\title{
The effect of familiarization on reading comprehension
}

College students $(\mathrm{N}=35)$ familiarized on paralogs which signified many of the important concepts and relationships in a story were able to answer correctly significantly more recall comprehension questions about the story than were subjects $(\mathrm{N}=33)$ who were familiarized on paralogs that were irrelevant to the story. The data indicated that the facilitation from the relevant familiarization was fairly uniform across the questions. The implications of the findings for teaching were briefly discussed.

Implicit in Ebbinghaus' invention of the CVC trigram in 1885 was the notion that previous familiarity with verbal materials facilitates the learning and retention of them. Since that time it has been well established that Ss familiarized with CVC trigrams learn serial lists of those materials, in rote fashion, more easily than they learn trigrams on which they were not previously familiarized (Nobel, 1963; Gillooly, 1966).

This study investigated the effect of familiarization, through repetitious pronouncing and copying of paralogs, on the comprehension of a story in which many of the important concepts and relationships of the story were signified by the paralogs themselves.

\section{Experimental Design}

The design of the experiment was a straightforward two group design in which comparison was made between the effects of relevant familiarization (RF) and irrelevant familiarization (IF) on the number of questions Ss answered correctly about a story based upon the relevant familiarized paralogs.

\section{Subjects}

Sixty-eight undergraduates who were enrolled in summer session courses in educational psychology and introductory psychology were assigned to RF and IF conditions by an unbiased procedure which yielded 33 in the IF group and 35 in the RF group.

\section{Materials}

Relevant and irrelevant noun and verb paralogs were taken from lists in Woodworth \& Schlosberg (1954, p. 703). The irrelevant noun paralogs were: $B A B A B$, DEFIG, FIMUR, NIGAT, POLEF, and ZUZUZ, and the irrelevant materials used as verbs were SARK(S), and THOG(GING). The relevant noun and verb paralogsGOKEN, KUPOD, LATUK, RUNIL, MEDON, TARUP, TWIC(S), and CRAD(DING)-occurred in the story below.

The tarup is a machine that was made by a scientist who liked baseball. He made the machine so that it could play baseball with him. The tarup can throw a ball with its medon and can catch a ball with its gokem. It can also pick up things from
FRANK B. MURRAY, UNIVERSITY OF MINNESOTA WLLIAM B. GILLOOLY, THE JOHNS HOPKINS UNIVERSITY

the ground with its gokem. It has two runils which tell it when a ball is coming so it can roll to catch it. The tarup can also hit a ball with a bat attached to its latuk. It never misses a ball that comes to it because it has two runils which always know where the ball is.

The tarup eats by cradding grass into little pieces during the day. When it has enough grass its kupod twics.

Would you like to have a tarup?

Below and on the same page as the story there was a labeled picture of the tarup (see Fig. 1).

The 10 comprehension questions, to which Ss responded with the correct missing term, were:

(1) The machine eats grass by the grass into little pieces.

(2) The catches the balls and picks things up from the ground.

(3) The machine the scientist made was called the

(4) The tells when the machine has had enough grass.

(5) The is used by the machine to throw balls.

(6) The machine can tell when the balls are coming because of its

(7) What does the machine do when it is full of grass? It

(8) The swings the bat.

(9) The gokem is on the same side of the machine as the

(10) The of the kupod here).

It should be noted that the information for correctly

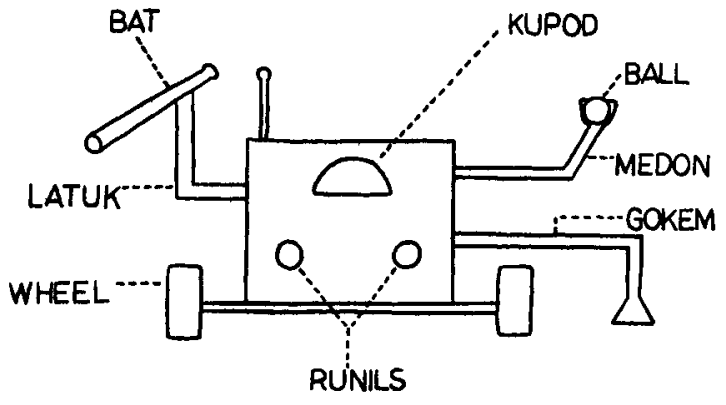

THIS IS A PICTURE OF THE TARUP

Fig. 1. Picture of tarup and labeling presented with story to Ss. 
Table 1. Chi-Square on Number of Correct Responses Between IF and RF Groups for Each Question

\begin{tabular}{ccccc} 
Question & IF $(\mathrm{N}=33)$ & $\mathrm{RF}(\mathrm{N}=35)$ & $x^{2}$ & $\mathrm{p}$ \\
\hline 1 & 16 & 31 & 12.74 & .001 \\
2 & 10 & 14 & .68 & $\mathrm{NS}$ \\
3 & 19 & 28 & 3.99 & .05 \\
4 & 4 & 13 & 7.99 & .01 \\
5 & 9 & 9 & .02 & $\mathrm{NS}$ \\
6 & 9 & 13 & .76 & $\mathrm{NS}$ \\
7 & 13 & 27 & 9.99 & .005 \\
8 & 7 & 15 & 3.64 & .10 \\
9 & 9 & 15 & 1.77 & $\mathrm{NS}$ \\
10 & 3 & 10 & 4.19 & .05 \\
\hline
\end{tabular}

answering the final two questions came solely from the diagram of the tarup.

The materials were collated into an IF booklet and a RF booklet of three pages: (1) the appropriate eight paralogs listed in six columns with one underlined space to the right of each paralog; (2) the tarup story and the picture; (3) the ten questions.

\section{Procedure}

After the two classes as groups were given the booklets, they were allowed 5 min to pronounce each paralog three times silently and copy it in the underlined space to the right of the paralog. By the end of the familiarization procedure, Ss had written each of the paralogs six times and pronounced it 18 times. Ss were then given $5 \mathrm{~min}$ to read the story about the tarup and to be prepared to answer questions about it. Finally, they were given $5 \mathrm{~min}$ to answer the 10 questions about the story. Criterial performance required spelling the paralog (answer) correctly.

\section{Results}

A one-tailed Mann-Whitney $U$ test indicated a significant difference between the IF and RF groups in the number of questions correctly answered $(z=3.06, p<$ $.001)$. The RF group answered more questions correctly (mean =6.54) than did the IF group (mean=5.06).

Cochran's $Q$ test indicated that for both the RF group $(Q=80.04$, df $=9, p<.001)$ and the IF group $(Q=52.50$, $\mathrm{df}=9, \mathrm{p}<.001$ ) the questions were of unequal difficulty. The correlation, corrected for ties, between the IF and RF groups with respect to question difficulty was high (rho $=.87, \mathrm{~N}=10, \mathrm{p}<.01$ ), indicating that although the questions were of unequal difficulty, they were so for both groups in approximately the same way. Apparently RF affected all the questions in a consistent fashion.

Correlations, corrected for ties, between the difficulty of questions and the number of times its answer appeared in the story, although negative for both groups, was only significant for the IF group (rho $=-.67, N=10$, $p<.05$, and for the RF group, rho $=-.53, N=10, p>.05)$. As one would expect, the degree of exposure to materials was more important to the IF group than to the RF group. A chi-square analysis of the differences between the IF and RF groups for each question indicated that differences were not always significant (Table1), but on no question was the IF group superior to the RF group.

Chi-square comparisons between questions (10) and (4) and between questions (9) and (5), which called for the same paralog answer when the information for one answer was in the story and when the information for the other was in the picture, indicated no significant differences in number of correct responses between questions in the IF and RF groups. The fact that the RF group was superior on questions (9) and (10), although insignificantly on (10), indicated that they learned more from the picture as well as more from the paragraph than did the IF group.

\section{Discussion}

The results of this experiment confirm the fact that familiarization can exert facilitative effects in a learning task more complex than that in which the facilitative effect was first discovered, namely the learning of a list of materials by rote. Whether the influence of familiarization extends beyond the mere imparting to students a vehicle by which they can emit their responses (that is, expressional fluency) in a task of this nature is a problem on which the authors are now working.

The data on question difficulty seem relevent to the problem of assessing the complexity of familiarization's influence. Although the questions used in this task were of unequal difficulty, the high agreement between the groups in the degree of difficulty associated with each question (rho $=.87$ ) indicated that familiarization did not interact with question difficulty. Apparently, the effect of familiarization is to impart a fairly uniform increase in performance across questions.

These results suggest two general situations in which extrinsic familiarization may be a useful pedagogical device: (1) when introducing typical children to novel terms in a subject matter (as in the type of situation investigated here), and (2) when introducing disadvantaged children to school-related words. More research on this issue may indicate that the educational advantages of the middle-class home are not solely due to the acquisition of information and concepts with which the school later deals. It may result in part from simply greater exposure to and familiarization with the terms ordinarily used to convey and organize knowledge.

\section{References}

Gillooly, W. B. The effect of familiarization instructions on associative latency and learning. Psychon. Sci., 1966, 4, 303-304.

Noble, C. E. Meaningfulness and familiarity. In C. N. Cofer \& B. S. Musgrave (Eds.), Verbal behavior and learning. New York: McGraw-Hill, 1963.

Woodworth, R. S., \& Schlosberg, H. Experimental psychology. New York: Holt, Rinehart \& Winston, 1954. 